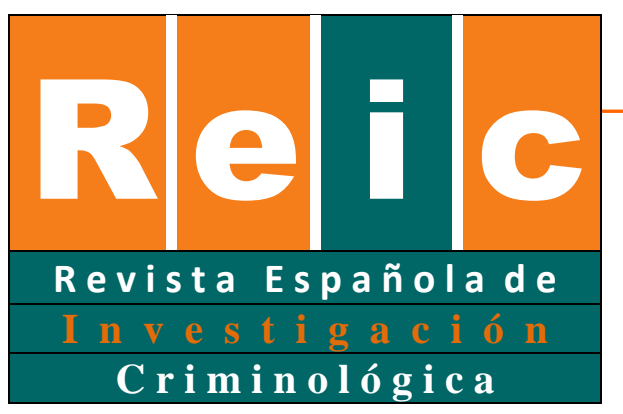

Aguilar

\title{
Diferencias entre feminicidios precedidos y no precedidos por la separación de la pareja
}

\section{Differences between femicides preceded or unpreceded by marital separation}

\author{
Raúl Aguilar Ruiz ${ }^{1}$ \\ Policía de la Generalitat-Mossos d'Esquadra
}

\begin{abstract}
RESUMEN
El conocimiento de las características de los distintos tipos de feminicidios puede contribuir en la mejora de las estrategias de prevención en el ámbito de la violencia contra la mujer. Con base en el análisis de 307 sentencias en España por feminicidios, consumados o intentados, se estudiaron las diferencias entre 146 feminicidios precedidos (FI-SE) y 161 no precedidos por la separación de la pareja (FI-NOSE). Los resultados muestran que los autores de FI-SE ejercen más tipos de violencia, son más celosos y experimentan más fuentes de estrés en el año que precede al crimen. El acoso incrementa cuatro veces la probabilidad de que ocurra el FI-SE, mientras que la discusión entre la pareja sin que conste violencia previa decrece la probabilidad de que acontezca el FI-SE. Sin embargo, los que cometen el FI-NOSE tienen un mayor historial de depresión y trastornos psicóticos. Se describen las implicaciones teóricas y prácticas.
\end{abstract}

Palabras clave: homicidio de pareja, feminicidio, factores de riesgo, violencia contra la pareja, separación.

\footnotetext{
${ }^{1}$ La correspondencia debe dirigirse a: Raúl Aguilar Ruiz, raguilarr@ uoc.edu

Revista Española de Investigación Criminológica

Artículo 6, Número 17 (2019)

https://doi.org/10.46381/reic.v17i0.167

Www.criminologia.net

ISSN: 1696-9219
} 


\begin{abstract}
Knowledge of the characteristics of the different types of femicides can contribute to the improvement of the prevention strategies in the field of violence against women. Based on the analysis of 307 sentences in Spain for femicide, be it actual or attempted, the differences between 146 femicides preceded by marital separation (FI-SE) and 161femicides not preceded by marital separation (FI-NOSE) were studied. The results show that the authors of FI-SE exert more types of violence against women, are more jealous, and experience more sources of stress. Stalking increases the probability that FI-SE occurs four times, while when the reason for aggression centers on an argument of the couple without any known previous violence, the probability that FI-SE occurs decreases. Nevertheless, those who commit FINOSE have a greater history of depression and psychotic disorders. Theoretical and practical implications are described.
\end{abstract}

Keywords: intimate partner homicide, femicide, risk factors, intimate partner violence, marital separation.

\title{
1. Introducción
}

En los últimos quince años (2003-2017) han muerto en España 923 mujeres en las relaciones de pareja, lo que representa una media aproximada de 61 víctimas por año. El homicidio en la pareja, a pesar de no ser un hecho exclusivo de los hombres sobre las mujeres, es cometido principalmente por hombres (CGPJ, 2018; Stöckl et al., 2013). En España se han producido avances muy importantes en la lucha contra la violencia sobre la mujer, entre los que destaca la Ley 27/2003, de 31 de julio, reguladora de la Orden de protección de las víctimas de la violencia doméstica, que incorporó un procedimiento sencillo y accesible de solicitar medidas de protección sin formalismos técnicos o costes añadidos. Por otro lado, la Ley Orgánica 1/2004, de 28 de diciembre, de Medidas de Protección Integral contra la Violencia de Género, supuso un impulso significativo en la mejora de la atención interinstitucional para luchar contra este problema de salud pública (OMS, 2002), reconociendo derechos como el de la información, la asistencia jurídica gratuita, la protección social y el apoyo económico. Por último, el Real Decreto-ley 9/2018, de 3 de agosto, de medidas urgentes para el desarrollo del Pacto de Estado contra la violencia de género, amplia los títulos judiciales habilitantes para acreditar la condición de víctima de violencia de género no supeditando la protección

Revista Española de Investigación Criminológica

Artículo 6, Número 17 (2019)

https://doi.org/10.46381/reic.v17i0.167

www.criminologia.net

ISSN: 1696-9219 
de estas mujeres al ejercicio de acciones legales como la denuncia, así como mejora la protección de los menores, hijas e hijos de las mujeres que viven en un contexto de maltrato.

Hoy se sabe que los perjuicios de padecer esta clase de violencia extrema no afectan solo a las mujeres, sino también a los hijos que sufren el ataque mortal (Alisic, Groot, Snetselaar, Stroeken \& van de Putte, 2015) o que han de afrontar las consecuencias potencialmente traumáticas de una doble pérdida. Por un lado, la de la madre. Por el otro, el usual suicidio o encarcelamiento del padre durante un largo periodo de tiempo (Ferrara et al., 2015). Por estos motivos, es necesario continuar indagando sobre los determinantes de este crimen con el objetivo de anticipar su ocurrencia, en aquellos casos en los que sea posible (Andrés-Pueyo, 2015).

\subsection{Evaluación del riesgo de feminicidio}

El hecho de considerar el feminicidio como una elección racional, más o menos condicionada por factores emocionales, contextuales y situacionales, constituye la base de la investigación empírica en aras de identificar los factores de riesgo asociados a la ocurrencia del comportamiento violento (Andrés-Pueyo \& Echeburúa, 2010; Hart, 2001). Un campo de estudio exitoso que ha permitido conocer qué factores discriminan entre la violencia en la pareja letal y la no letal (Cunha \& Gonçalves, 2016; Dobash, Dobash, Cavanagh \& MedinaAriza, 2007; Echeburúa, Fernández-Montalvo \& de Corral, 2008), así como crear guías de evaluación del riesgo de feminicidio (Campbell, Webster \& Glass, 2009; Echeburúa, Fernández-Montalvo, de Corral \& López-Goñi, 2009; Wang, 2015).

Sin embargo, parece que estos instrumentos han llegado al límite de su capacidad predictiva (Monahan \& Skeem, 2014; Muñoz-Vicente \& López-Ossorio, 2016). Además, la reincidencia delictiva como medida clásica de evaluación de la predicción de estas herramientas se ve seriamente comprometida en el caso del feminicidio, ya que los niveles mayores de riesgo conllevan medidas más intensas de protección (Loinaz, 2017, p.74). Asimismo, la concepción de que todos aquellos que matan a su pareja o expareja son un grupo homogéneo (Kivisto, 2015) o que todo el maltrato contra la mujer es igual, afecta negativamente a la prevención y gestión del riesgo, pues imposibilita aplicar estrategias 
adaptadas a cada tipo particular de violencia (Gulliver \& Fanslow, 2015; Kelly \& Johnson, 2008).

Tampoco existe un patrón claro y homogéneo que permita prever cuándo ocurrirá el ataque mortal. Hay homicidios que suceden de manera explosiva en el contexto de una discusión, mientras que otros se enmarcan en un ambiente de conflictividad creciente en torno a la ruptura sentimental o la supuesta infidelidad de la mujer (Fernández Teruelo, 2011; Gnisci \& Pace, 2016). A pesar de que en el comportamiento delictivo están implicados diferentes factores que influyen en interacción sobre las personas (Redondo, 2015), el campo de la evaluación del riesgo está limitado en la comprensión de las características inmediatas o factores agudos que provocan que un individuo cometa la acción criminal (Serin, Chadwick \& Lloyd, 2016; Sheehan, Murphy, Moynihan, Dudley-Fennessey \& Stapleton, 2015).

Un aspecto que podría mejorar la comprensión y, por consiguiente, la prevención del feminicidio es la investigación intragrupo. Es decir, indagar en las posibles taxonomías de homicidios en la pareja y qué factores de riesgo se asocian a cada una de ellas (Dixon, Hamilton-Giachritsis \& Browne, 2008; López-Ossorio et al., 2018).

\subsection{Determinantes del feminicidio}

En cuanto al historial delictivo y psicopatología de los feminicidas los resultados no son concluyentes. Hay quien sostiene que estos maltratadores se asemejan más al perfil del homicida o al delincuente violento en general, con rasgos antisociales de personalidad y alteraciones mentales (Eke, Hilton, Harris, Rice \& Houghton, 2011; Loinaz, Marzabal \& Andrés-Pueyo, 2018; Thomas, Dichter \& Matejkowski, 2011), mientras que otros afirman que se parecen más al hombre normalizado o convencional, sin patología mental destacable (Dobash \& Dobash, 2011).

En lo que respecta a las conductas de maltrato que perpetran los feminicidas contra la mujer, las revisiones de la literatura y meta-análisis revelan que las más usuales son la agresión física, las amenazas, el estrangulamiento, la tenencia de armas de fuego, la conducta suicida, la escalada de la violencia, el acoso, las agresiones sexuales, el control y los celos (Breu, Guggenbichler \& Wollmann, 2008; Campbell, Glass, Sharps, Laughon \& Bloom, 
2007; Spencer \& Stith, 2018). No obstante, no todos los feminicidas ejercen todos estos tipos de violencia (Nicolaidis et al., 2003).

También se han identificado las principales motivaciones vinculadas a estos crímenes. Entre ellas destacan el abandono o el anuncio de romper la relación afectiva por parte de la víctima, así como los celos (Adinkrah, 2014; CGPJ, 2018, p.61; Dobash \& Dobash, 2011; Fernández Teruelo, 2013). Sin embargo, aunque con una menor prevalencia, se enumeran otras motivaciones como el trastorno mental grave o los conflictos en la pareja sin que existan antecedentes previos de maltrato (Adinkrah, 2014; Johnson, Eriksson, Mazerolle \& Wortley, en prensa; Flynn, Gask, Appleby \& Shaw, 2016; Juodis, Starzomski, Porter \& Woodworth, 2014; Nicolaidis et al., 2003).

Por último, se ha estudiado la influencia de distintas fuentes de estrés como las dificultades económicas, la pérdida del empleo, la denuncia, la emisión de una orden de protección, la infidelidad de la pareja, los problemas legales, así como la enfermedad física o psíquica grave, en la comisión del feminicidio. Algunos investigadores han hallado que la infidelidad, las denuncias y las hospitalizaciones por trastornos mentales graves se asocian al feminicidio (Eriksson, 2013; Kerry, 2001), mientras que otros no han encontrado relación entre el feminicidio y la denuncia previa de la mujer (Sanz-Barbero, Heras-Mosterio, OteroGarcía \& Vives-Cases, 2016). En cuanto a los factores ambientales, incluso se ha llegado a estudiar la influencia del aumento de la temperatura ambiental en la comisión del feminicidio (Sanz-Barbero et al., 2018).

\subsection{Diferencias entre feminicidios precedidos y no precedidos por la separación de la pareja}

Un estudio a fondo del proceso de separación podría ser útil para comprender mejor este crimen (Dobash et al., 2007), ya que las intervenciones de aquellos que trabajan para prevenir la violencia contra la mujer podrían ser efectivas cuando la pareja está conviviendo, pero dejar de serlo en el caso de que ya se hubieran separado (Ellis, Stuckless \& Smith, 2014). Sin embargo, todavía se desconocen las diferencias existentes entre los feminicidios íntimos precedidos por la separación de la pareja (FI-SE) y aquellos que ocurren mientras la pareja 
mantiene la relación afectiva (FI-NOSE) (Gnisci \& Pace, 2016; Goussinsky \& YassourBorochowitz, 2012).

En la investigación se asume que la dinámica subyacente a la violencia cometida por parejas o exparejas es la misma, pero los procesos etiológicos que originan y perpetúan la agresión en cada situación poseen una dinámica propia (Ellis \& Stuckless, 2006). De hecho, se ha constatado que la violencia entre exparejas puede llegar a ser incluso más severa que la cometida entre aquellos que no se han separado (Reckdenwald \& Simone, 2017). En el mismo sentido, considerando que el abandono del agresor es un factor de riesgo de feminicidio, no se ha abordado suficientemente lo que provoca que esta decisión sea especialmente provocadora para algunos hombres (Goussinsky \& Yassour-Borochowitz, 2012).

Por los motivos anteriormente expuestos, el objetivo principal de esta investigación es conocer las diferencias entre el feminicidio íntimo precedido por la separación de la pareja (FI-SE) y el no precedido por la separación en el año previo al crimen (FI-NOSE) en los grupos de factores siguientes: a) historial delictivo y psicopatología, b) tipos de violencia en la pareja, c) motivación criminal y fuentes de estrés. El objetivo secundario pretende revelar qué factores de riesgo predicen el FI-SE. Las variables asociadas al historial delictivo y psicopatología, así como las correspondientes al tipo de violencia en la pareja son habituales en el establecimiento de tipologías de maltratadores a partir de la propuesta ya clásica de Holtzworth-Munroe y Stuart (1994). Las variables que componen la motivación criminal y fuentes de estrés se han seleccionado atendiendo a lo propuesto por algunas investigaciones en aras de identificar aquellos factores más dinámicos y agudos que desencadenan el feminicidio (Aguilar, 2018; Eriksson, 2013).

Las hipótesis derivadas de los dos objetivos son:

$\mathrm{H}_{1}$ : hay diferencias entre el FI-SE y el FI-NOSE en el historial delictivo y psicopatología.

$\mathrm{H}_{2}$ : hay diferencias entre el FI-SE y el FI-NOSE en los tipos de violencia en la pareja. $\mathrm{H}_{3}$ : hay diferencias entre el FI-SE y el FI-NOSE en la motivación criminal y fuentes de estrés.

$\mathrm{H}_{4}$ : existen predictores del FI-SE versus el FI-NOSE. 


\section{Método}

\subsection{Muestra}

En esta investigación retrospectiva de caso control se analizaron 307 sentencias dictadas por las Audiencias Provinciales en España entre el año 2012 y el 2015 por feminicidios en la pareja (FI), consumados o en grado de tentativa. La muestra se dividió en dos grupos. El primero $(n=146)$ estuvo compuesto por feminicidios precedidos por el abandono de la mujer en el año previo al crimen (FI-SE). En el segundo $(n=161)$, se incluyeron los feminicidios no precedidos por la separación de la pareja en el año previo al feminicidio (FI-NOSE). La muestra seleccionada representa aproximadamente el $92 \%$ de todas las sentencias.

\subsection{Instrumento}

Se analizó la información recogida en las sentencias mediante una hoja de registro creada ad hoc. El documento consta de 30 variables. La primera relativa al tipo de feminicidio (FI-NOSE=0; FI-SE=1). Las 29 restantes corresponden a las variables objeto de estudio agrupadas en los tres factores siguientes: A) Historial delictivo y psicopatológico del agresor: 2) antecedentes penales, 3) abuso de alcohol o drogas, 4) posesión de armas de fuego, 5) trastornos mentales, y 6) trastornos de personalidad; B) Tipo de violencia contra la mujer ejercida durante la relación: 7) maltrato físico, 8) agresión con armas u objetos, 9) estrangulamiento, 10) agresiones sexuales, 11) amenazas de muerte, 12) amenazas de suicidio, 13) quebrantamientos de medidas de alejamiento, 14) control y celos, 15) acoso en el año previo, y 16) escalada de la violencia; C) Motivación criminal y fuentes de estrés en el último año: 17) celos, 18) discusión, 19) establecimiento de una nueva relación, 20) denuncias, 21) problemas legales, 22) disputas por la custodia de hijos, 23) económicos, 24) enfermedad física del agresor, 25) enfermedad psíquica del agresor, y 26) enfermedad física o psíquica de la víctima. Estas variables son categóricas y su nivel de medida es nominal $(0=$ no consta; $1=$ sí consta).

Revista Española de Investigación Criminológica

Artículo 6, Número 17 (2019)

www.criminologia.net

ISSN: 1696-9219 
Por otro lado, a partir de las variables anteriores se crearon cuatro nuevas variables métricas. La variable 27) "suma de trastorno mental" compuesta a partir de la suma de los trastornos mentales. La variable 28) "suma de trastornos de personalidad", formada por la suma de este tipo de trastornos. La variable 29) "suma de tipos de violencia", a partir del sumatorio de todas las clases de violencia en la pareja. Por último, la variable 30) "suma de estrés", resultado del sumatorio de todas las fuentes de estrés. La creación de variables compuestas a partir del sumatorio de fuentes de estrés o tensión y variables respecto al tipo de violencia contra la mujer es un hecho recomendado y usualmente empleado en la investigación (Eriksson, 2013; Hardesty et al., 2015). El nivel de medida de estas variables es de escala.

Las variables objeto de estudio se eligieron con base en la literatura sobre el riesgo de feminicidio y sobre tipologías de maltratadores (Aguilar, 2018; Campbell et al., 2007; CGPJ, 2018; Eriksson, 2013; Holtzworth-Munroe \& Stuart, 1994; Kerry, 2001; Kivisto, 2015).

\subsection{Procedimiento}

En el 2016 se inició la investigación. En primer lugar, se seleccionó la muestra. Según el Instituto Nacional de Estadística, entre el 2012 y el 2015 se dictaron en España 332 sentencias judiciales por feminicidios, incluyendo tanto las tentativas como los consumados. El hecho de analizar conjuntamente los feminicidios consumados y tentados se debe a que, en ambos casos, según el artículo 16 de la Ley Orgánica 10/1995, de 23 de noviembre, del Código Penal, el autor de los hechos quiso matar a la persona, lo único que en el feminicidio en grado de tentativa no logró su propósito por causas independientes de su voluntad. Se buscaron las 332 sentencias en el Centro de Documentación Judicial (CENDOJ) del CGPJ. Para ello se cumplimentaron los campos de búsqueda de la base de datos de la siguiente manera: Jurisdicción= penal; Tipo de órgano= Audiencia Provincial; Tipo de resolución= todas; Fecha de resolución= entre 2012 y 2015; Texto libre $=$ homicidio $\mathrm{O}$ asesinato $\mathrm{Y}$ mujer. La muestra inicial fue de 5759 sentencias. A continuación, se leyó el contenido de cada resolución judicial. Se excluyeron las sentencias por feminicidios imprudentes, las que hacían referencia a una relación homosexual, las duplicadas y aquellas en las que el autor de 
los hechos fue una mujer. Se obtuvieron 307 sentencias que cumplían los criterios de inclusión, 161 por feminicidios consumados y 146 por tentados, lo que representa el $92.5 \%$ de todas las sentencias. Para dividir los casos en los dos grupos a comparar se aplicó en siguiente criterio. Las sentencias asignadas al primer grupo (FI-SE) ( $n=146 ; 47.6 \%)$, fueron aquellas en las que constaba que la víctima había abandonado o comunicado en alguna ocasión a su pareja su intención de salir de la relación en el año previo al feminicidio. En el grupo de control (FI-NOSE) $(n=161 ; 52.4 \%)$ se incluyeron las sentencias en las que la mujer no había abandonado o comunicado esta intención a su pareja en el año previo al feminicidio.

\subsection{Análisis estadísticos}

Se utilizaron técnicas estadísticas descriptivas univariantes (frecuencias) para describir la muestra y bivariantes para los contrastes de independencia en las variables de estudio entre el grupo de FI-SE y el de FI-NOSE. Para el contraste de independencia entre variables categóricas se utilizó la prueba chi-cuadrado. No obstante, en los supuestos en los que no se cumplió el requisito de esta técnica, que exige que al menos el $80 \%$ de los valores esperados en las celdas sean mayores que 5, se utilizó la prueba exacta de Fisher. Respecto a las variables numéricas, dada la no normalidad de la distribución, se utilizó la prueba no paramétrica $U$ de Mann-Whitney. Se estableció un nivel de confianza del $95 \%$ bilateral. Asimismo, para medir la magnitud de la asociación en aquellos casos en que se hallaron diferencias significativas, se utilizó el coeficiente $P h i$ de Cramer en las variables categóricas y la Correlación biserial puntual $R b p$ en las métricas.

Posteriormente, para conocer qué variables predecían el FI-SE versus el FI-NOSE se llevó a cabo la prueba multivariante de la regresión logística binaria. La variable dependiente fue el tipo de feminicidio (FI-SE= 1; FI-NOSE =0). Las variables independientes que se introdujeron en el modelo de regresión fueron las que mostraron diferencias significativas en la prueba de contraste chi-cuadrado y $U$ de Mann-Whitney. Para introducir las variables métricas en la prueba de regresión, se transformaron en variables nominales dicotómicas. En particular, "suma de trastorno mental" se transformó en dicotómica ( 0 trastornos mentales= no trastornos mentales; 1, 2, 3, 4 o 5= sí trastornos mentales). "Suma de tipos de violencia"

\section{Revista Española de Investigación Criminológica}


se transformó en dicotómica ( 0,1 tipo de violencia= pocos tipos de violencia; 2, 3, 4, 5, 6, 5 tipos de violencia= muchos tipos de violencia). Finalmente, "suma de estrés", se transformó en dicotómica ( 0 estresores $=$ estrés bajo; 1, 2, 3, 4 estresores $=$ estrés elevado). Para evaluar la fiabilidad interjueces, dos observadores revisaron un $30 \%$ de las sentencias obteniéndose un grado de acuerdo fuerte según el índice Kappa de Cohen $(K=.81 ; p<.01)$. El análisis se realizó con el software SPSS (versión 21).

\section{Resultados}

\subsection{Características más relevantes de la muestra}

De los 307 feminicidios, el 52.4\% fueron consumados y $47.6 \%$ en grado de tentativa. En cuanto a las características criminológicas de los agresores, el $22.8 \%$ tenía antecedentes penales y el $23.8 \%$ abuso de sustancias. Los trastornos mentales más frecuentes fueron los relacionados con sustancias (8.1\%), los psicóticos (8.1\%), los adaptativos (4.9\%) y el depresivo (4.6\%). Asimismo, el tipo de maltrato más usual fue la agresión física (31.6\%), las amenazas de muerte (23.8\%), el acoso (21.5\%), así como los celos y las conductas de control (19.5\%). El 47.6\% de los casos el feminicidio estuvo asociado a la separación de la pareja. En el 17.9\% destacaron los celos como motivo del crimen y en el 13\% los conflictos entre la pareja. Por último, las tres fuentes principales de estrés en el año precedente al feminicidio fueron la denuncia (19.2\%), el establecimiento de una nueva relación afectiva de la mujer con otro hombre $(17.3 \%)$ y los problemas económicos $(8.8 \%)$.

\subsection{Diferencias entre el FI-SE y el FI-NOSE en el historial delictivo y psicopatológico}

Se constató una mayor posesión de armas de fuego en los FI-SE en comparación con los FI-NOSE, $8.9 \%$ vs. 1.9\%, $p=.006$ (Tabla 1). Sin embargo, no hubo diferencias ni en los antecedentes penales, $25.3 \%$ vs. $20.5 \%$, ns, ni en el abuso de consumo de alcohol o drogas, $21.2 \%$ vs. $20.5 \%$, ns. Por otro lado, respecto al trastorno mental, los autores de FI-NOSE 
tenían más antecedentes por trastornos psicóticos, $12.4 \%$ vs. 3.4\%, p=.004, y depresión, $7.5 \%$ vs. $1.4 \%, p=.011$. No obstante, no se hallaron diferencias en otros trastornos mentales ni en ningún trastorno de personalidad. Finalmente, la suma total de trastornos mentales fue mayor en el grupo de FI-NOSE, $U=10240.50, p=.009$.

Tabla 1.

Diferencias entre el FI-SE y el FI-NOSE en el historial delictivo y psicopatológico del agresor

\begin{tabular}{|c|c|c|c|c|c|}
\hline Variables & $\begin{array}{c}\text { FI-NOSE } \\
\begin{array}{c}(n=161) \\
n(\%)\end{array}\end{array}$ & $\begin{array}{c}\text { FI-SE } \\
(n=146) \\
n(\%)\end{array}$ & $X^{2}$ & $g l$ & Phi \\
\hline 2) Antecedentes penales & $33(20.5)$ & $37(25.3)$ & 1.02 & 1 & ns \\
\hline 3) Abuso alcohol o drogas & $42(26.1)$ & $31(21.2)$ & .99 & 1 & ns \\
\hline 4) Posesión arma de fuego & $3(1.9)$ & $13(8.9)$ & $7.68 * *$ & 1 & .16 \\
\hline \multicolumn{6}{|l|}{ 5) Trastornos mentales } \\
\hline Tm. Orgánicos & $2(1.2)$ & $2(1.4)$ & $.01 \mathrm{a}$ & 1 & ns \\
\hline Tm. Consumo psicotrópicos & $13(8.1)$ & $12(8.2)$ & .00 & 1 & ns \\
\hline Tm. Psicóticos & $20(12.4)$ & $5(3.4)$ & $8.28 * *$ & 1 & -.16 \\
\hline Tm. Ansiedad & $8 \quad(5.0)$ & $2(1.4)$ & $3.14 \mathrm{a}$ & 1 & ns \\
\hline Tm. Depresión & $12(7.5)$ & $2(1.4)$ & $6.51^{*}$ & 1 & -.15 \\
\hline Tm. Adaptativos & $5 \quad(3.2)$ & $10(6.8)$ & 2.30 & 1 & $n s$ \\
\hline \multicolumn{6}{|l|}{ 6) Trastornos de personalidad } \\
\hline Tp. Paranoide & $\begin{array}{ll}1 & (.6)\end{array}$ & $1 \quad(.7)$ & $.01 \mathrm{a}$ & 1 & ns \\
\hline Tp. Disocial & $1 \quad(.6)$ & $4(2.7)$ & $2.15 \mathrm{a}$ & 1 & $n s$ \\
\hline Tp. Ansioso & $\begin{array}{ll}1 & (.6)\end{array}$ & $0 \quad(.0)$ & $.91 \mathrm{a}$ & 1 & $n s$ \\
\hline Tp. Dependiente & $\begin{array}{ll}1 & (.6)\end{array}$ & $0 \quad(.0)$ & $.91 \mathrm{a}$ & 1 & $n s$ \\
\hline \multicolumn{2}{|r|}{$M(D E)$} & \multirow{2}{*}{$M(D E)$} & $\boldsymbol{U}$ & \multicolumn{2}{|r|}{$r b p$} \\
\hline 27) Suma de trastornos mentales & $.37(.62)$ & & $10240.50 * *$ & & .17 \\
\hline $\begin{array}{l}\text { 28) Suma de trastornos } \\
\text { personalidad }\end{array}$ & $.06 \quad(.24)$ & $.07(.32)$ & 11672.00 & & ns \\
\hline
\end{tabular}

Notas. FI-NOSE = feminicidio no precedido por la separación. FI-SE= feminicidio precedido por la separación. $\mathrm{a}=$ Prueba de Fisher. $n s=$ no diferencias significativas.

$* p<.05 . * * p<.01$. 


\subsection{Diferencias entre el FI-SE y el FI-NOSE en el tipo de violencia ejercida contra la mujer en la relación de pareja}

Los autores de FI-SE cometieron más tipos de maltrato que los de FI-NOSE (Tabla 2). En particular, se registraron más agresiones con armas, $11 \%$ vs. 3.7\%, $p=.014$, amenazas de muerte, $38.8 \%$ vs. $10.6 \%, p<.001$, amenazas de suicidio, $7.5 \%$ vs. $2.5 \%, p=.040$, y quebrantamientos de condena, $24.7 \%$ vs. $14.9 \%, p<.001$. En este sentido, también se halló una mayor presencia de agresión física sin armas en los FI-SE, pero sin llegar a alcanzar la significación estadística, 36.3\% vs. 27.3\%, ns. Asimismo, los FI-SE se mostraron más celosos y controladores, $24.7 \%$ vs. $14.9 \%, p=.031$, acosaron más a la mujer en el último año, $37.7 \%$ vs. $6.8 \%, p<.001$, e incrementaron la escalada de violencia, $50.7 \%$ vs. $16.1 \%, p<.001$. Para acabar, los FI-SE presentaron un índice mayor en la suma de tipos de violencia contra la mujer, $U=7563, p<.001$.

Tabla 2.

Diferencias entre el FI-SE y el FI-NOSE en el tipo de violencia ejercida contra la mujer en la relación de pareja

\begin{tabular}{|c|c|c|c|c|c|}
\hline Variables & $\begin{array}{c}\text { FI-NOSE } \\
\begin{array}{c}(n=161) \\
n(\%)\end{array}\end{array}$ & $\begin{array}{c}\text { FI-SE } \\
(n=146) \\
n(\%)\end{array}$ & $X^{2}$ & $g l$ & Phi \\
\hline 7) Maltrato físico & $44(27.3)$ & $53(36.3)$ & 2.85 & 1 & $n s$ \\
\hline 8) Agresión con armas & $6(3.7)$ & $16(11.0)$ & $6.02 *$ & 1 & .14 \\
\hline 9) Estrangulamiento & $1 \quad(.6)$ & $4 \quad(2.7)$ & $2.14 \mathrm{a}$ & 1 & $n s$ \\
\hline 10) Agresión sexual & $2(1.2)$ & $4 \quad(2.7)$ & $0.89 \mathrm{a}$ & 1 & $n s$ \\
\hline 11) Amenazas de muerte & $17(10.6)$ & $56(38.4)$ & $32.64 * * *$ & 1 & .33 \\
\hline 12) Amenazas de suicidio & $4 \quad(2.5)$ & $11 \quad(7.5)$ & $4.20^{*}$ & 1 & .12 \\
\hline 13) Quebrantamiento medida alejamiento & $13(8.1)$ & $36(24.7)$ & $15.69 * * *$ & 1 & .23 \\
\hline 14) Control y celos & $24(14.9)$ & $36(24.7)$ & $4.63 *$ & 1 & .12 \\
\hline 15) Acoso año previo & $11(6.8)$ & $55(37.7)$ & $43.14 * * *$ & 1 & .38 \\
\hline 16) Escalada violencia & $26(16.1)$ & $74(50.7)$ & $41.58 * * *$ & 1 & .37 \\
\hline \multicolumn{2}{|r|}{$M(D E)$} & $M(D E)$ & \multicolumn{2}{|l|}{$\boldsymbol{U}$} & $r b p$ \\
\hline 29) Suma de tipos de violencia & $.87(1.32)$ & $2.09(2.01)$ & $7563.00 * * *$ & & .35 \\
\hline
\end{tabular}

Notas. FI-NOSE = feminicidio no precedido por la separación. FI-SE= feminicidio precedido por la separación. $\mathrm{a}=$ Prueba de Fisher. $n s=$ no diferencias significativas.

$* p<.05 . * * p<.01 . * * * p<.001$. 


\subsection{Diferencias entre el FI-SE y el FI-NOSE en la motivación y en las fuentes de estrés en el año precedente al feminicidio}

Por otra parte, los celos, como motivo criminal, estuvo más presente en los autores de FI-SE, 22.6\% vs. 13.7\%, $p=.041$, mientras que las discusiones en la pareja sin que constaran antecedentes de violencia previa se dieron en más ocasiones en los FI-NOSE, $22.4 \%$ vs. 2.7\%, $p<.001$ (Tabla 3). Asimismo, los FI-SE experimentaron un índice global más elevado de estrés durante el año que antecedió al crimen, $U=9699, p=.003$. En particular, los FI-SE habían sido denunciados en más ocasiones, $30.1 \%$ vs. $9.3 \%, p<.001$, experimentaron más problemas legales y judiciales, $6.8 \%$ vs. $1.9 \%, p=.030$, y tuvieron conocimiento o creyeron que su mujer había comenzado una nueva relación sentimental con otro hombre, $21.9 \%$ vs. $13 \%, p=.040$. En cambio, el hecho de sufrir en el año previo al feminicidio un nuevo trastorno mental grave o de padecer un empeoramiento del estado psíquico se constató más en los FI-NOSE, $10.6 \%$ vs. $0 \%, p<.001$.

\section{Tabla 3.}

Diferencias entre el FI-SE y el FI-NOSE en la motivación y en las fuentes de estrés en el año precedente al feminicidio

\begin{tabular}{|c|c|c|c|c|c|}
\hline Variables & $\begin{array}{c}\text { FI-NOSE } \\
\begin{array}{c}(n=161) \\
n(\%)\end{array}\end{array}$ & $\begin{array}{c}\text { FI-SE } \\
(n=146) \\
n(\%)\end{array}$ & $X^{2}$ & $g l$ & Phi \\
\hline \multicolumn{6}{|l|}{ Motivo del homicidio } \\
\hline 17)Celos & $22(13.7)$ & $33(22.6)$ & $4.16^{*}$ & 1 & .12 \\
\hline 18)Discusión sin violencia previa & $36(22.4)$ & $4 \quad(2.7)$ & $26.01 * * *$ & 1 & -.29 \\
\hline \multicolumn{6}{|l|}{ Fuentes de estrés } \\
\hline 19)Nueva pareja de la mujer & $21(13.0)$ & $32(21.9)$ & $4.22 *$ & 1 & .12 \\
\hline 20)Ella denuncia & $15 \quad(9.3)$ & $44(30.1)$ & $21.37 * * *$ & 1 & .26 \\
\hline 21)Problemas legales & $3 \quad(1.9)$ & $10 \quad(6.8)$ & $4.69^{*}$ & 1 & .12 \\
\hline 22)Disputas por custodia & $8 \quad(5.0)$ & $14 \quad(9.6)$ & 2.46 & 1 & $n s$ \\
\hline 23)Económicos & $13 \quad(8.1)$ & $14 \quad(9.6)$ & .22 & 1 & $n s$ \\
\hline 24)Enfermedad física de él & $1 \quad(0.6)$ & $1 \quad(.7)$ & $.01 \mathrm{a}$ & 1 & $n s$ \\
\hline
\end{tabular}

Revista Española de Investigación Criminológica 


\begin{tabular}{|c|c|c|c|c|c|}
\hline 25)Enfermedad psíquica de él & $17(10.6)$ & $0 \quad(.0)$ & $16.60 * * *$ & 1 & -.23 \\
\hline 26) Enfermedad de ella & $5 \quad(3.1)$ & $(.0)$ & $4.61 \mathrm{a}$ & 1 & $n s$ \\
\hline \multicolumn{2}{|r|}{$M(D E)$} & $M(D E)$ & $\boldsymbol{U}$ & & $r b p$ \\
\hline 30)Suma de estrés en el año previo & $.52(.70)$ & $.79(.85)$ & $9699.00 * *$ & & .18 \\
\hline
\end{tabular}

Notas. FI-NOSE = feminicidio no precedido por la separación. FI-SE= feminicidio precedido por la separación. $\mathrm{a}=$ Prueba de Fisher. $n s=$ no diferencias significativas.

$* p<.05 .{ }^{* *} p<.01 . * * * p<.001$.

Sobre las demás fuentes de estrés, no se encontraron diferencias entre grupos en la afectación derivada de los procesos vinculados a la custodia de los hijos, $9.6 \%$ vs. $5 \%$, ns, en enfermedades físicas del agresor, $0.7 \%$ vs. $0.6 \%$, ns, o de la mujer, $0 \%$ vs. $3.1 \%$, ns, ni en los problemas económicos, $9.6 \%$ vs. $8.1 \%, n s$.

\subsection{Factores de riesgo predictores de FI-SE vs. FI-NOSE}

Se realizó la prueba de regresión logística para identificar los factores predictores del FI-SE (Tabla 4). En el modelo de regresión se introdujeron las variables que resultaron estadísticamente significativas en los contrastes de independencia. El modelo fue significativo, $X^{2}(19)=117.546, p<.001$. En función de -cuadrado, entre el $31.8 \%$ (Cox y Snell) y 42.4\% (Nagelkerke) de la varianza se explicó por el conjunto de las variables. El modelo clasificó correctamente el $73.9 \%$ de los casos. De las variables introducidas, dos de ellas contribuyeron significativamente al modelo. Concretamente, el acoso, $\mathrm{OR}=4.209 ; 95$ $\mathrm{CI}=[1.383,12.808]$, y la discusión entre la pareja como motivo del crimen, OR=.160; 95 $\mathrm{CI}=[.052, .492]$. En concreto, los que cometen el feminicidio en el contexto de la separación tienen 4 veces más probabilidades de haber acosado a la pareja en el año previo al crimen. Por el contrario, la probabilidad de perpetrar el feminicidio en un contexto de ruptura afectiva disminuye si el motivo principal del homicidio gira en torno a discusiones y disputas en la pareja sin que conste la existencia de un maltrato previo contra la mujer. 


\section{Tabla 4.}

Factores de riesgo de FI-SE vs. FI-NOSE

\begin{tabular}{|c|c|c|c|c|c|c|c|c|}
\hline \multirow{2}{*}{ Variables predictoras } & \multirow{2}{*}{$\boldsymbol{B}$} & \multirow{2}{*}{$E S$} & \multirow{2}{*}{ Wald } & \multirow{2}{*}{$g l$} & \multirow{2}{*}{$p$} & \multirow{2}{*}{ OR } & \multicolumn{2}{|c|}{$95 \%$ IC } \\
\hline & & & & & & & $\boldsymbol{L I}$ & $L S$ \\
\hline Posesión arma de fuego & 1.328 & .747 & 3.161 & 1 & .075 & 3.773 & .873 & 16.303 \\
\hline Suma trastorno mental & .089 & .426 & .044 & 1 & .834 & 1.094 & .475 & 2.520 \\
\hline Tm. Psicóticos & -.239 & .818 & .085 & 1 & .771 & .788 & .159 & 3.913 \\
\hline Tm. Depresión & -1.320 & .936 & 1.988 & 1 & .159 & .267 & .043 & 1.673 \\
\hline Suma tipos de violencia & -.546 & .580 & .888 & 1 & .346 & .579 & .186 & 1.804 \\
\hline Agresión con armas & 1.002 & 672 & 2.224 & 1 & .136 & 2.725 & .730 & 10.174 \\
\hline Amenazas de muerte & .755 & .446 & 2.871 & 1 & .090 & 2.128 & .888 & 5.100 \\
\hline Amenazas suicidas & 1.136 & .762 & 2.225 & 1 & .136 & 3.115 & .700 & 13.864 \\
\hline Quebrantamiento medidas & .713 & .573 & 1.547 & 1 & .214 & 2.040 & .663 & 6.276 \\
\hline Controlador / celoso & -.311 & .463 & .451 & 1 & .502 & .733 & .296 & 1.816 \\
\hline Acoso año previo & 1.437 & .568 & 6.408 & 1 & $.011 *$ & 4.209 & 1.383 & 12.808 \\
\hline Escalada violencia & .310 & .585 & .281 & 1 & .596 & 1.364 & .433 & 4.294 \\
\hline Motivo celos & -.656 & .648 & 1.025 & 1 & .311 & .519 & .146 & 1.847 \\
\hline Motivo discusión & -1.834 & .574 & 10.210 & 1 & $.001 * *$ & .160 & .052 & .492 \\
\hline Suma de estrés & -.166 & .440 & .143 & 1 & .706 & .847 & .357 & 2.007 \\
\hline Otra pareja & 1.168 & .732 & 2.543 & 1 & .111 & 3.215 & .765 & 13.500 \\
\hline Ella denuncia & .355 & .574 & .382 & 1 & .537 & 1.426 & .463 & 4.394 \\
\hline Problemas legales & 1.312 & .875 & 2.248 & 1 & .134 & 3.714 & .668 & 20.641 \\
\hline Enfermedad psíquica de él & -21.436 & 9087.307 & .000 & 1 & .998 & .000 & .000 & \\
\hline Constante & -.406 & .226 & 3.226 & 1 & .072 & .666 & & \\
\hline
\end{tabular}

Notas. FI-NOSE = feminicidio no precedido por la separación. FI-SE= feminicidio precedido por la separación. $\mathrm{ES}=$ error estándar; $\mathrm{OR}=$ Odds Ratio; $\mathrm{IC}=$ intervalos de confianza; $\mathrm{LI}=$ límite inferior; $\mathrm{LS}=$ límite superior. Variable dependiente $=$ Tipo de feminicidio $(0=$ FI-NOSE; $1=$ FI-SE $)$.

$-2 \log$ verosimilitud $=307.313 ; R^{2}=.318$ (Cox y Snell); .424 (Nagelkerke). $N=307 . X^{2}(19)=117.546, p<.001$. Hosmer y Lemeshow $=\mathrm{X}^{2}(8)=4.023, p=.855$; Predicción del modelo $=73.9 \%$ correcto.

$*_{p}<.05 . * * p<.01$. 


\section{Discusión}

En función de los resultados, existen diferencias entre el FI-SE y el FI-NOSE en los tres grupos de factores de estudio (a, b y c), aunque no en todas las variables, de lo cual se deriva una confirmación solo parcial de las hipótesis alternativas planteadas.

En cuanto al historial delictivo y psicopatológico, a pesar de que uno de cada cuatro o cinco maltratadores tiene antecedentes penales y abusa de las sustancias no hay diferencias entre los dos grupos. No obstante, aunque la presencia de alteraciones mentales es baja, la depresión, los trastornos psicóticos y el número de trastornos mentales es superior en los FINOSE. Por otro lado, los autores de FI-SE ejercen más modalidades de maltrato. De hecho, ninguno de los tipos de violencia estudiados está más presente en los FI-NOSE. Los FI-SE están sometidos a un mayor número de fuentes de estrés y consuman el feminicidio en más ocasiones por motivos de celos, mientras que en los FI-NOSE hay una mayor presencia de enfermedad mental y conflictos entre la pareja sin que haya conocimiento de violencia previa.

Los FI-SE comparten rasgos de dos categorías distintas de feminicidas. En primer lugar, se asemejan a los denominados "maltratadores crónicos" (Kivisto, 2015), a los "homicidas antisociales y violentos en general" (Aguilar, 2017; Dixon et al., 2008) en el historial delictivo, abuso de sustancias, así como en la gravedad de la violencia contra la mujer. No obstante, en cuanto a la experimentación de celos y las consecuencias derivadas de la separación, los FI-SE también se parecen a los "disfóricos borderline o sobrecontrolados" (Dixon et al., 2008; Kivisto, 2015).

Otro factor destacable es que los tipos de maltrato más presentes en los FI-SE (agresiones con armas u objetos, amenazas de muerte y de suicidio, las conductas de acoso, el abandono, la escalada de la violencia, el control y los celos, así como los quebrantamientos de las medidas de protección judiciales) suelen figurar como factores de riesgo en las guías de evaluación del riesgo de violencia letal y casi letal (Campbell et al., 2009; Echeburúa, Amor, Loinaz \& de Corral, 2010; Wang, 2015).

Los autores de FI-SE también experimentan más estresores en los meses próximos al crimen, destacándose entre las principales fuentes de estrés la denuncia, los problemas legales y judiciales, así como el conocimiento de una nueva relación íntima de la pareja con 
otro hombre. Estos datos están en consonancia con los alcanzados en otras investigaciones llevadas a cabo en países como África, Canadá o Australia (Adinkrah, 2008; Breu et al., 2008; Eriksson, 2013).

Respecto a los que cometen el FI-NOSE, algunos de estos maltratadores se asemejan a la tipología del "enfermo mental". Estos hombres padecen trastornos mentales graves con sintomatología psicótica, tienen un historial menor de violencia previa contra la pareja y abuso de substancias, y su motivación criminal no está vinculada a los celos o al abandono (Aguilar, 2018; Kivisto, 2015). Otro aspecto significativo de esta investigación es que la probabilidad de que acontezca un FI-SE disminuye si la motivación criminal gira en torno a los conflictos en la pareja sin que consten episodios de maltrato previos.

Esta clase de violencia conocida por el nombre de "conflictos mutuos" (Kelly \& Johnson, 2008) también ha sido encontrada en los feminicidios (Goussinsky \& YassourBorochowitz, 2012; Johnson et al., en prensa). Ampliando este aspecto, Nicolaidis et al. (2003), a raíz de entrevistas con víctimas supervivientes de un intento de feminicidio, advirtieron que los factores de riesgo variaban mucho en cada caso. De hecho, el 17\% de las víctimas describieron episodios de violencia menor que serían difíciles de categorizar como maltrato y el $7 \%$ no sufrieron agresiones físicas ni conductas de control.

El único estresor en el año previo al crimen que afecta más a los FI-NOSE en comparación con los FI-SE es el hecho de sufrir un nuevo trastorno mental grave o experimentar un episodio agudo de crisis mental. En la misma línea, Flynn et al. (2016) advirtieron, con base en el estudio de 60 homicidios-suicidios en Inglaterra y Gales, que el $12 \%$ de los homicidas había contactado en los doce meses previos al crimen con los servicios de salud mental y el $7 \%$ lo hizo un mes antes.

En cuanto a los factores predictores del FI-SE, se destaca la relevancia del acoso como fuerte predictor del feminicidio en el contexto de la ruptura sentimental, siendo este un hecho también detectado en otras investigaciones. Moracco, Runyan y Butts (1998) registraron acoso en el 23\% de las mujeres que fueron asesinadas. Por su parte, McFarlane et al. (1999) informaron que aquellas mujeres que fueron seguidas o espiadas tuvieron cuatro veces más probabilidades de sufrir un ataque letal; un índice de acoso que fue superior en las que habían abandonado al agresor respecto a las que todavía convivían cuando fueron atacadas. 
Relativo a la prevención, los hallazgos de esta investigación permiten inferir dos escenarios de riesgo distintos para cada tipo de feminicidio. En los FI-SE se advierte un grado de violencia mayor contra la mujer y un aumento de las conductas de acoso meses antes del feminicidio. También están sometidos a un mayor número de estresores derivados de la situación de la separación. En suma, el riesgo de que se produzca el feminicidio en estos supuestos presentaría un mejor pronóstico de detección, ya que en el periodo que antecede al crimen acontecen eventos que pueden alertar al profesional que evalúa el caso sobre la existencia de una situación potencialmente peligrosa.

Ahondando en los FI-SE, el $47.6 \%$ de las mujeres padecieron un ataque letal o casi letal durante el año siguiente a haber abandonado a su pareja. Puesto que el riesgo de ser asesinada se incrementa en el momento de separarse del agresor, continúa siendo elevado en los dos o tres meses posteriores, y decrece a partir del año (Ellis et al., 2014), es importante establecer medidas de protección de la mujer adecuadas para ayudarla a afrontar el proceso de ruptura sentimental (Campbell et al., 2003).

Por otro lado, la predicción y prevención de los FI-NOSE se complica a causa de la ausencia de un encadenamiento claro de factores que permitan anticipar el crimen. Algunos de estos feminicidios se originan en un contexto de conflictos en la pareja sin que existan antecedentes conocidos de violencia previa, mientras que otros son perpetrados por hombres que presentan un menor historial delictivo, abuso de sustancias, celos y maltrato. A excepción del padecimiento de una crisis mental aguda, que afecta solo a uno de cada diez hombres, no hay otros desencadenantes agudos o una escalada progresiva de la violencia que alerte del incremento del riesgo de feminicidio.

Profundizando en la patología mental grave, prever cuando acontecerá el asesinato en estos casos es difícil, pues en las investigaciones sobre taxonomías de feminicidas se concluye que los homicidios en el contexto de la enfermedad mental apenas comparten los factores de riesgo usuales que figuran en las guías diseñadas para predecir la ocurrencia de estos crímenes (Aguilar, 2018; Kivisto, 2015). Por este motivo, el riesgo de feminicidio no puede ser detectado atendiendo exclusivamente al uso de estas herramientas. No obstante, el hecho de que algunos hombres que sufren depresión o delirios (Flynn et al., 2016), especialmente si giran en torno al perjuicio de su mujer (SAP Barcelona 154/2014, de 12 de 
febrero), suelan acudir a los servicios de salud mental, tendría que ser considerado por los profesionales de la salud.

En lo que concierne a la investigación futura, es fundamental continuar indagando en las motivaciones subyacentes y en las fuentes de estrés que condicionan el feminicidio. Así, mientras que las preguntas cuánto, cómo y por quién han sido investigadas, por qué y bajo qué circunstancias permanecen relativamente sin respuesta (Goussinsky \& YassourBorochowitz, 2012). De hecho, se postula que no solo es importante aquello que ocurre, sino cuando acaece, lo que comporta considerar este crimen como un fenómeno derivado de la interacción entre factores personales, relacionales y situacionales (Gnisci \& Pace, 2016; Sebire, 2017). Así, referente a la intervención y prevención, qué y cuándo podría funcionar mejor dependerá de quién esté bajo consideración y en qué lugar se encuentre en el camino que conduce al feminicidio (Juodis et al., 2014). En este aspecto, este trabajo supone un avance en la línea de investigación poco explorada entre los distintos tipos de feminicidios, integrando variables individuales, tipo de violencia en la relación de pareja, motivacionales y factores de estrés.

Respecto a las limitaciones de este trabajo, las sentencias analizadas no representan a los feminicidas que se suicidan después del asesinato, pues en estos casos no se dicta ninguna sentencia. Según el CGPJ (2016) se suicidan en España aproximadamente el $20 \%$ de los feminicidas. En futuras investigaciones, las entrevistas en profundidad con mujeres supervivientes, familiares o amigos, podría mejorar la comprensión de los factores asociados al feminicidio-suicidio. Sobre esta cuestión, en España existe un equipo nacional de revisión pormenorizada de homicidios de pareja que, inspirado en equipos similares que ya trabajan en otros países desde hace años, busca conocer en profundidad las causas de este fenómeno criminal (González et al., 2018). 


\section{Referencias}

Adinkrah, M. (2008). Husbands Who Kill Their Wives. International Journal of Offender Therapy and Comparative Criminology, 52(3), 296-310. https://doi.org/10.1177/0306624X07307119

Adinkrah, M. (2014). Intimate partner femicide-suicides in Ghana: Victims, offenders, and incident characteristics. Violence Against Women, 20(9), 1078-1096. https://doi.org/10.1177/1077801214549637

Aguilar, R. (2017). El feminicidio. Diferencias entre el homicida antisocial y el normalizado. Boletín Criminológico, 4, 1-12. http://dx.doi.org/10.24310/Boletincriminologico.2017.v23i0.3876

Aguilar, R. (2018). Tipologías de Feminicidas con Trastorno Mental en España. Anuario de Psicología Jurídica, 28(1), 39-48. http://dx.doi.org/10.5093/apj2018a4

Alisic, E., Groot, A., Snetselaar, H., Stroeken, T.\& van de Putte, E. (2015). Parental intimate partner homicide and its consequences for children: protocol for a population-based study. BMC Psychiatry, 15(1), 177. https://doi.org/10.1186/s12888-015-0565-z

Andrés-Pueyo, A. (2015). (29 de agosto de 2015). Feminicidios: qué hacer. [Mensaje en un Blog]. $\quad$ Retrieved from http://laspersonasonlaclave.blogspot.com.es/2015/08/feminicidios2017-quehacer.html

Andrés-Pueyo, A. \& Echeburúa, E. (2010). Valoración del riesgo de violencia: Instrumentos disponibles e indicaciones de aplicación. Psicothema, 22(3), 403-409. Retrieved from http://www.psicothema.com/pdf/3744.pdf

Breu, F., Guggenbichler, S. \& Wollmann, J. (2008). Woman killing: Intimate femicide in Ontario, 1974-1994. Resources for Feminist Research, 26, 151-174.

Campbell, J. C., Glass, N., Sharps, P. W., Laughon, K. \& Bloom, T. (2007). Intimate Partner Homicide. Trauma, Violence, \& Abuse, 8(3), 246-269. https://doi.org/10.1177/1524838007303505

Campbell, J. C., Webster, D., Koziol-McLain, J., Block, C., Campbell, D., Curry, M. A., ... Laughon, K. (2003). Risk Factors for Femicide in Abusive Relationships: Results From a Multisite Case Control Study. American Journal of Public Health, 93(7), 1089-1097. https://doi.org/10.2105/AJPH.93.7.1089

Campbell, J. C., Webster, D. W. \& Glass, N. (2009). The Danger Dssessment: Validation of a lethality risk assessment instrument for intimate partner femicide. Journal of Interpersonal Violence, 24(4), 653-674. https://doi.org/10.1177/0886260508317180

Consejo General del Poder Judicial. (2016). Informe sobre las víctimas mortales de la violencia de género y de la violencia doméstica en el ámbito de la pareja o la expareja en 2014. Madrid: Consejo General del Poder Judicial.

Consejo General del Poder Judicial. (2018). Análisis de las sentencias dictadas en el año 2016, relativas a homicidios y/o asesinatos entre los miembros de la pareja o expareja $y$ de menores a manos de sus progenitores. Madrid: Consejo General del Poder Judicial. 
Cunha, O. S. \& Gonçalves, R. A. (2016). Severe and Less Severe Intimate Partner Violence: From Characterization to Prediction. Violence and Victims, 31(2), 235-250. https://doi.org/10.1891/0886-6708.VV-D-14-00033

Dixon, L., Hamilton-Giachritsis, C. \& Browne, K. (2008). Classifying Partner Femicide. Journal of Interpersonal Violence, 23(1), 74-93. https://doi.org/10.1177/0886260507307652

Dobash, R. E. \& Dobash, R. P. (2011). What Were They Thinking? Men Who Murder an Intimate Partner. Violence Against Women, 17(1), 111-134. https://doi.org/10.1177/1077801210391219

Dobash, R. E., Dobash, R. P., Cavanagh, K. \& Medina-Ariza, J. (2007). Lethal and Nonlethal Violence Against an Intimate Female Partner. Violence Against Women, 13(4), 329353. https://doi.org/10.1177/1077801207299204

Echeburúa, E., Amor, P. J., Loinaz, I. \& de Corral, P. (2010). Escala de Predicción del Riesgo de Violencia Grave contra la pareja -Revisada- (EPV-R). Psicothema, 22(4), 10541060. https://doi.org/10.1037/t14533-000

Echeburúa, E., Fernández-Montalvo, J. \& de Corral, P. (2008). ¿Hay diferencias entre la violencia grave y la violencia menos grave contra la pareja?: Un análisis comparativo. International Journal of Clinical and Health Psychology, 8(2), 355-382.

Echeburúa, E., Fernández-Montalvo, J., de Corral, P. \& López-Goñi, J. J. (2009). Assessing Risk Markers in Intimate Partner Femicide and Severe Violence. Journal of Interpersonal Violence, 24(6), 925-939. https://doi.org/10.1177/0886260508319370

Eke, A. W., Hilton, N. Z., Harris, G. T., Rice, M. E. \& Houghton, R. E. (2011). Intimate Partner Homicide: Risk Assessment and Prospects for Prediction. Journal of Family Violence, 26(3), 211-216. https://doi.org/10.1007/s10896-010-9356-y

Ellis, D. \& Stuckless, N. (2006). Separation, domestic violence, and divorce mediation. Conflict Resolution Quarterly, 23(4), 461-485. https://doi.org/10.1002/crq.150

Ellis, D., Stuckless, N. \& Smith, C. (2014). Marital separation and lethal domestic violence. New York: Routledge.

Eriksson, L. (2013). Male perpetrators of intimate partner homicide: a general strain theory explanation (doctoral dissertation). Griffith University, Brisbane. Retrieved from https://www120.secure.griffith.edu.au/rch/items/8c970962-ff78-414a-a7f9 c6f88d9ed756/1/

Fernández Teruelo, J. G. (2011). Feminicidios de género: evolución real del fenómeno, el suicidio del agresor y la incidencia del tratamiento mediático. Revista Española de Investigación Criminológica, 9, 1-27. Retrieved from https://reic.criminologia.net/index.php/journal/article/view/58

Fernández Teruelo, J. G. (2013). Riesgo de feminicidio de Género en situaciones de ruptura de la relación de pareja. Estudios Penales y Criminológicos, 33, 149-173. Retrieved from https://www.usc.es/revistas/index.php/epc/article/view/1253

Ferrara, P., Caporale, O., Cutrona, C., Sbordone, A., Amato, M., Spina, G., ... Scambia, G. (2015). Femicide and murdered women's children: which future for these children orphans of a living parent? Italian Journal of Pediatrics, 41(1), 68. https://doi.org/10.1186/s13052-015-0173-z 
Flynn, S., Gask, L., Appleby, L. \& Shaw, J. (2016). Homicide - suicide and the role of mental disorder: a national consecutive case series. Social Psychiatry and Psychiatric Epidemiology, 51(6), 877-884. https://doi.org/10.1007/s00127-016-1209-4

Gnisci, A. \& Pace, A. (2016). Lethal domestic violence as a sequential process: Beyond the traditional regression approach to risk factors. Current Sociology, 64(7), 1108-1123. https://doi.org/10.1177/0011392116629809

González, J. L., Garrido, M. J., López, J. J., Muñoz, J. M., Arribas, A., Carbajosa, P. \& Ballano, E. (2018). Revisión Pormenorizada de Homicidios de Mujeres en las Relaciones de Pareja en España. Anuario de Psicología Jurídica, 28(1), 28-38. https://doi.org/10.5093/apj2018a2

Goussinsky, R. \& Yassour-Borochowitz, D. (2012). "I killed her, but I never laid a finger on her" - A phenomenological difference between wife-killing and wife-battering. Aggression and Violent Behavior, 17(6), 553-564. https://doi.org/10.1016/j.avb.2012.07.009

Gulliver, P. \& Fanslow, J. L. (2015). The Johnson typologies of intimate partner violence: An investigation of their representation in a general population of New Zealand women. Journal of Child Custody, 12(1), 25-46. https://doi.org/10.1080/15379418.2015.1037051

Hardesty, J. L., Crossman, K. A., Haselschwerdt, M. L., Raffaelli, M., Ogolsky, B. G. \& Johnson, M. P. (2015). Toward a standard approach to operationalizing coercive control and classifying violence types. Journal of Marriage and Family, 77(4), 833843. https://doi.org/10.1111/jomf.12201

Hart, S. (2001). Assessing and managing violence risk. En Douglas K. S. (Eds.), HCR-20, violence risk Management companion guide (pp. 13-26).Vancouver: SFU Editors.

Holtzworth-Munroe, A. \& Stuart, G. L. (1994). Typologies of male batterers: Three subtypes and the differences among them. Psychological Bulletin, 116, 476-497. https://doi.org/10.1037/0033-2909.116.3.476

Johnson, H., Eriksson, L., Mazerolle, P. \& Wortley, R. (en prensa). Intimate femicide: The role of coercive control. Feminist Criminology, 155708511770157. https://doi.org/10.1177/1557085117701574

Juodis, M., Starzomski, A., Porter, S. \& Woodworth, M. (2014). A Comparison of domestic and non-domestic homicides: further evidence for distinct dynamics and heterogeneity of domestic homicide perpetrators. Journal of Family Violence, 29(3), 299-313. https://doi.org/10.1007/s10896-014-9583-8

Kelly, J. B. \& Johnson, M. P. (2008). Differentiation among types of intimate partner violence: research update and implications for interventions. Family Court Review, 46(3), 476-499. https://doi.org/10.1111/j.1744-1617.2008.00215.x

Kerry, G. (2001). Understanding and predicting intimate femicide: An analysis of men who kill their intimate female partners. (doctoral dissertation). Carleton University, Ottawa, Canada.

Kivisto, A. J. (2015). Male Perpetrators of Intimate Partner Homicide: A Review and Proposed Typology. The Journal of the American Academy of Psychiatry and the Law, 43(3), 300-312. Retrieved from http://www.ncbi.nlm.nih.gov/pubmed/26438808 
Loinaz, I. (2017). Manual de evaluación del riesgo de violencia. Metodología y ámbitos de aplicación. Madrid: Pirámide.

Loinaz, I., Marzabal, I. \& Andrés-Pueyo, A. (2018). Risk factors of female intimate partner and non-intimate partner homicides. European journal of psychology applied to legal context, 10. 49-55. https://doi.org/10.5093/ejpalc2018a4

López-Ossorio, J. J., Carbajosa, P., Cerezo-Domínguez, A. I., González-Álvarez, J. L., Loinaz, I. \& Muñoz-Vicente, J. M. (2018). Taxonomía de los homicidios de mujeres en las relaciones de pareja. Psychosocial Intervention, 27(2), 95-104. https://doi.org/10.5093/pi2018a11

McFarlane, J. M., Campbell, J. C., Wilt, S., Sachs, C., Ulrich, Y. \& Xu, X. (1999). Stalking and Intimate Partner Femicide. Homicide Studies, 3(4), 300-316. https://doi.org/10.1177/1088767999003004003

Monahan, J. \& Skeem, J. L. (2014). The evolution of violence risk assessment. CNS Spectrums, 19(5), 419-424.

Moracco, K. E., Runyan, C. W. \& Butts, J. D. (1998). Femicide in North Carolina, 19911993: A statewide study of patterns and precursors. Homicide Studies, 2(4), 422-446.

Muñoz-Vicente, J. M. \& López-Ossorio, J. J. (2016). Valoración psicológica del riesgo de violencia: alcance y limitaciones para su uso en el contexto forense. Anuario de Psicología Jurídica, 26(1), 130-140. https://doi.org/10.1016/j.apj.2016.04.005

Nicolaidis, C., Curry, M. A., Ulrich, Y., Sharps, P., McFarlane, J., Campbell, D., ... Campbell, J. (2003). Could we have known? A qualitative analysis of data from women who survived an attempted homicide by an intimate partner. Journal of General Internal Medicine, 18(10), 788-794. https://doi.org/10.1046/j.15251497.2003.21202.x

OMS. (2002). Informe mundial sobre la violencia y la salud. Washington, DC: Organización Mundial de la Salud.

Redondo, S. (2015). El origen de los delitos. Introducción al estudio y explicación de la criminalidad. Valencia: Tirant lo Blanch.

Reckdenwald, A. \& Simone, S. (2017). Injury patterns for homicide followed by suicide by the relationship between victims and offenders. Homicide Studies, 21(2), 111-132. https://doi.org/10.1177/1088767916671350

Sanz-Barbero, B., Heras-Mosterio, J., Otero-García, L. \& Vives-Cases, C. (2016). Perfil sociodemográfico del feminicidio en España y su relación con las denuncias por violencia de pareja. Gaceta Sanitaria, 30(4), 272-278. https://doi.org/10.1016/j.gaceta.2016.03.004

Sanz-Barbero, B., Linares, C., Vives-Cases, C., González, J. L., López-Ossorio, J. J. \& Díaz, J. (2018). Heat wave and the risk of intimate partner violence. Science of the Total Environment, 644, 413-419. https://doi.org/10.1016/j.scitotenv.2018.06.368

Sebire, J. (2017). The value of incorporating measures of relationship concordance when constructing profiles of intimate partner homicides: A descriptive study of IPH committed within London, 1998-2009. Journal of interpersonal violence, 32(10), $1476-1500$ 
Serin, R. C., Chadwick, N. \& Lloyd, C. D. (2016). Dynamic risk and protective factors. Psychology, Crime \& Law, 22(1-2), 151-170. https://doi.org/10.1080/1068316X.2015.1112013

Sheehan, B. E., Murphy, S. B., Moynihan, M. M., Dudley-Fennessey, E. \& Stapleton, J. G. (2015). Intimate partner homicide: New insights for understanding lethality and risks. Violence Against Women, 21(2), 269-288. https://doi.org/10.1177/1077801214564687

Spencer, C. M. \& Stith, S. M. (2018). Risk factors for male perpetration and female victimization of intimate partner homicide: A meta-analysis. Trauma, Violence, and Abuse. Avance de publicación online. https://doi.org/10.1 177/1524838018781101

Stöckl, H., Devries, K., Rotstein, A., Abrahams, N., Campbell, J., Watts, C. \& Moreno, C. G. (2013). The global prevalence of intimate partner homicide: a systematic review. The Lancet, 382(9895), 859-865. https://doi.org/10.1016/S0140-6736(13)61030-2

Thomas, K. A., Dichter, M. E. \& Matejkowski, J. (2011). Intimate versus nonintimate partner murder: A comparison of offender and situational characteristics. Homicide Studies, 15(3), 291-311. https://doi.org/10.1177/1088767911417803

Wang, P. (2015). Assessing the Danger. Journal of Interpersonal Violence, 30(14), 24282446. https://doi.org/10.1177/0886260514553114

\section{Agradecimientos}

El autor quiere agradecer a Gerard Manyer y Mayte Saval, expertos en el ámbito de la atención y protección de víctimas, sus aportaciones respecto a la concreción de variables relevantes objeto de estudio y su contribución en el análisis de casos.

Raúl Aguilar Ruiz es subinspector de policía, jefe de una unidad responsable del área de protección y atención a las víctimas. Licenciado en psicología y criminología. Experto en perfilación criminal. Profesor del Institut de Seguretat Pública de Cataluña de la materia sobre valoración del riesgo de violencia. Ha formado a profesionales de la psicología, trabajo social, salud, derecho y otros servicios especializados en violencia contra la mujer, en la prevención e intervención en casos de alto riesgo de feminicidio. 